\title{
Grupos de apoio para homens com câncer de próstata: revisão integrativa da literatura
}

\author{
Support groups for prostate cancer patients: \\ integrative literature review
}

Murilo dos Santos Moscheta ${ }^{1}$

Manoel Antônio dos Santos ${ }^{2}$

${ }^{1}$ Departamento de

Psicologia. Universidade

Estadual de Maringá.

Av. Colombo 5790, Zona 07. 87020-900 Maringá PR. murilomoscheta@me.com

${ }^{2}$ Faculdade de Filosofia, Ciências e Letras de Ribeirão Preto, Universidade de São Paulo.
Abstract This article is an integrative literature review to analyze the contribution of national and international studies examining the use of psychological support groups as a care strategy for patients diagnosed with prostate cancer. The electronic databases consulted were: LILACS, MedLine and PsycINFO, over a period of 20 years (1989-2009) with pre-defined criteria for inclusion. Eleven studies were selected, and based on their contributions some considerations are presented regarding the factors that favor male participation in the support groups and the potential benefits of such participation; as well as their main concerns and the influence of gender identity in the process of coping with illness. Furthermore, studies suggest some models of group intervention, along with orientation for the coordinators which emphasize the importance of these groups as an effective strategy to aid in the process of coping of prostate cancer patients.

Key words Prostatic neoplasms, Self-help groups, Social support, Literature review as a topic
Resumo Este artigo caracteriza-se como uma revisão integrativa da literatura, que teve como objetivo analisar a contribuição dos estudos nacionais e internacionais acerca do uso de grupos de apoio psicológico como estratégia de cuidado de pacientes diagnosticados com câncer de próstata. Foram consultadas as bases eletrônicas: Lilacs, MedLine e PsycINFO, em intervalo de 20 anos (1989-2009), com critérios de inclusão preestabelecidos. Foram selecionados 11 estudos, cujas evidências permitiram tecer considerações acerca dos fatores que favorecem a participação de homens nos grupos de apoio, dos potenciais beneficios dessa participação, das principais preocupações que eles manifestam e a influência da identidade de gênero no processo de enfrentamento da doença. Além disso, os estudos apontam para alguns modelos de intervenção em grupo, oferecem orientações aos coordenadores para sua condução e salientam a importância de se implementar grupos como uma estratégia efetiva de cuidado aos homens que enfrentam o câncer de próstata. Palavras-chave Neoplasias da próstata, Grupos de auto-ajuda, Apoio Social, Literatura de revisão como assunto 


\section{Introdução}

O câncer de próstata constitui hoje um problema de saúde em nível mundial, tendo aumentado sua incidência desde a década de 60 . Atualmente, responde por cerca de $12 \%$ das causas de mortalidade em todo o mundo ${ }^{1}$. O número de casos novos de câncer de próstata estimado para o Brasil, no ano de 2010, é de 52.350. Estes valores correspondem a um risco estimado de 54 casos novos a cada 100 mil homens. É o tipo de câncer mais comum entre a população masculina brasileira, seguido das neoplasias de pulmão, estômago, cólon e reto ${ }^{2}$. Devido à maior prevalência em idosos, o câncer de próstata constitui uma preocupação de saúde muito importante quando se considera o significativo aumento da expectativa de vida da população ${ }^{3}$.

Apesar de ser o tipo mais comum de câncer entre os homens, é também o mais difícil de ser discutido, limitando suas possibilidades de cuidado. Fatores socioculturais, incluindo os estereótipos de gênero, crenças e valores que definem o que é ser masculino, têm sido apontados como obstáculos na implementação de práticas de cuidado em saúde. Assim o exame do toque retal, embora bastante eficaz quando combinado com o exame de sangue na deteç̧ão precoce do tumor de próstata, ainda é relativamente pouco realizado, possivelmente por esbarrar em preconceitos relacionados aos estereótipos de gênero $^{4,5}$. Este cenário conduz à alta prevalência de diagnóstico de câncer de próstata em estadiamento avançado e, infelizmente, com piores prognósticos. Atento a esta questão, no contexto nacional, o Ministério da Saúde lançou, em 2008, a Política Nacional de Atenção Integral à Saúde do Homem $^{6}$ como uma das prioridades de governo, reconhecendo que os agravos do sexo masculino constituem verdadeiros problemas de saúde pública.

Nas diferentes fases de enfrentamento da doença, desde o diagnóstico, passando pelo tratamento e culminando com a reabilitação psicossocial, o paciente com câncer de próstata tem sua vida alterada em dimensões físicas, psíquicas e sociais. Durante o diagnóstico, por exemplo, é comum que a o paciente sinta-se estigmatizado uma vez que, no imaginário coletivo, a doença é frequentemente associada à morte. Além disso, o câncer de próstata afeta especificamente uma localização anatômica responsável pelas funções sexuais do homem e tem o potencial de desencadear uma série de conflitos ligados à sua sexualidade. Na fase de tratamento, destacam-se as di- ficuldades decorrentes das intervenções terapêuticas que em geral incluem a prostatectomia radical, a quimioterapia e a radioterapia. Não é raro que ao término destes procedimentos o paciente tenha que lidar com sequelas como diminuição da libido, impotência sexual e incontinência urinária. Assim, o câncer de próstata é uma doença que determina um importante impacto no cotidiano dos homens afetados, em geral resultando em mudanças drásticas em suas vidas.

Estudos mostram que a rede de apoio social é um importante recurso para o enfrentamento destas mudanças ${ }^{7}$. A família e a rede de apoio social existente previamente ao aparecimento da doença, são destacados como as principais fontes de apoio. Segundo a literatura, estes contextos relacionais auxiliam o paciente a se adaptar aos rigores do tratamento, reduzem o estresse, favorecem o incremento da qualidade de vida e até mesmo contribuem no prolongamento da sobrevida ${ }^{7}$. Levando-se isto em conta, grupos de apoio psicológico têm sido propostos como alternativa suplementar para aqueles pacientes $\mathrm{cu}$ jas relações familiares e sociais, por alguma razão, não se configuram com a força necessária para o amparo de suas necessidades.

\section{Grupo de apoio no contexto da saúde}

Os grupos de apoio têm apresentado uma demanda crescente no contexto nacional e internacional e vêm se consolidando como uma modalidade de cuidado eficaz ${ }^{7}$. No contexto da saúde, têm ampla utilização e se organizam de forma bastante variada. De modo geral, pode-se dizer que podem ser constituídos dentro dos contextos dos equipamentos de saúde, como hospitais e unidades básicas de saúde, ou no âmbito da comunidade, em associações de bairros ou grupos sociais organizados. Podem ser coordenados por um especialista, em geral algum profissional com formação em grupoterapia ou coordenação de grupo, ou por alguém que viva em condições semelhantes às da população para a qual o grupo se dirige. Quanto à sua sistemática de trabalho, tais grupos podem ser homogêneos ou heterogêneos, abertos ou fechados, de frequência semanal, quinzenal ou mensal, com duração variável e objetivos múltiplos. Graças a essa diversidade, nota-se uma infinidade de arranjos grupais desenhados para atender as necessidades de populações específicas.

Em geral, os participantes buscam obter informações, orientações, opiniões, encorajamento, compartilhamento de experiências e apoio 
psicológico. Esses grupos oferecem um espaço no qual é possível desenvolver habilidades de enfrentamento da doença e seu tratamento, a partir do contato com outras pessoas que vivenciam situações semelhantes. Essa conexão entre pessoas com interesses afins é entendida como um poderoso recurso empregado na reabilitação física e psíquica dos pacientes ${ }^{8}$.

$\mathrm{Na}$ área da saúde ainda são poucas as publicações que permitem dimensionar a amplitude dos aspectos envolvidos no uso da estratégia de grupos de apoio para o cuidado do paciente com câncer de próstata. Considerando-se a relevância desse conhecimento para a sistematização do cuidado, este trabalho teve como objetivo analisar a contribuição dos estudos nacionais e internacionais acerca da aplicação de grupos de apoio psicológico como estratégia de cuidado de pacientes diagnosticados com câncer de próstata.

\section{Método}

Este estudo caracteriza-se como uma revisão integrativa da literatura ${ }^{9}$. A qual supõe que, mediante a delimitação de uma questão de pesquisa, o investigador deve voltar-se para o processo de exploração rigorosa e padronizada da literatura, abrindo caminho para análise e discussão da produção científica da área, de modo a promover conhecimento mais aprofundado do fenômeno estudado ${ }^{10,11}$. A questão que norteou a presente investigação foi: qual é a contribuição dos grupos de apoio psicológico no cuidado de homens com câncer de próstata?

O método de investigação fundamentado na revisão integrativa busca manter os padrões de clareza, rigor e replicação dos estudos primários. Assim, para a realização da revisão, além da formulação de questões norteadoras e hipóteses, é necessário estabelecer critérios claros de inclusão e exclusão para composição da amostra, definição de características dos estudos primários levantados, análise de dados coligidos, interpretação dos resultados obtidos e, finalmente, apresentação da revisão ${ }^{11}$.

Para realizar o levantamento bibliográfico foram selecionados os descritores disponíveis na lista Health Science Descriptors / Medical Subject Headings (DeCS/MeSH), a saber: "neoplasias da próstata" e "grupos de autoajuda", seus sinônimos em língua portuguesa "câncer de próstata" e "grupos de apoio", e seus equivalentes nos idiomas inglês e espanhol. Esses descritores foram utilizados para busca nas seguintes bases de da- dos bibliográficos: Lilacs (Literatura Latino-americana e do Caribe), MedLine (United States National Library of Medicine) e PsycINFO (base de dados da American Psychological Association). As bases indexadoras foram escolhidas por concentrarem a maior parte das publicações de impacto na interface da psicologia e das ciências da saúde.

Foram considerados os seguintes parâmetros limitadores da busca inicial: a) artigos publicados entre 1989 e 2009; b) redigidos em língua portuguesa, espanhola ou inglesa; e, c) publicações que tivessem como foco os grupos de apoio para pacientes com câncer de próstata. Foram excluídas as publicações que não estivessem em formato de artigo científico, como livros, teses, dissertações, resenhas, cartas e editoriais, ou que não estavam disponíveis na íntegra para acesso online pelo portal de periódicos da Capes. Esta escolha se deve ao fato de que, em uma das bases utilizadas (Lilacs), notou-se o registro de trabalhos nessas modalidades. São estudos que, muitas vezes, podem não ter sido submetidos a um rigoroso processo de avaliação. A revisão por pares (peer review) é uma auditoria de qualidade realizada às cegas por outros pesquisadores, que é tradição em publicações científicas. Garante a qualidade do artigo e a sua obediência aos padrões de rigor científico que devem nortear sua apreciação. A fim de realçar apenas os trabalhos submetidos a esse processo rigoroso de avaliação, optou-se por selecionar exclusivamente artigos indexados.

Após levantamento preliminar nas bases de dados escolhidas, os resumos dos artigos selecionados foram revisados de modo a se poder refinar a escolha final das publicações que comporiam o corpus deste estudo. Nesta etapa foram excluídos os artigos que não satisfaziam os critérios de inclusão referidos ou que: a) tratavam de grupos de apoio de natureza não-psicológica, como, por exemplo, grupos de reabilitação física; b) enfocavam os aspectos comunicacionais e relacionais entre paciente e equipe em contextos extragrupais; c) utilizavam-se do grupo como fonte de recrutamento para pesquisa ou como espaço de coleta de dados, mas não tinham o grupo em si como objeto da investigação ou como parte do objetivo do estudo.

Após a aplicação dos critérios de inclusão e exclusão e o refinamento posterior da busca, o corpus desta revisão terminou constituído de 11 artigos qualificados, que foram lidos e catalogados segundo uma ficha que resume seus atributos gerais. Nessa ficha foram identificados: a) base de dados consultada; b) autores; c) dados refe- 
rentes à publicação (ano, periódico, país de origem, idioma); d) objetivos do estudo; e) metodologia empregada; e, f) resultados e discussão ${ }^{11}$.

Os resultados foram sistematizados de maneira a ressaltar as seguintes dimensões: a) identificar, de modo sistematizado, as publicações nacionais e internacionais sobre grupos de apoio psicológico no contexto da assistência ao câncer de próstata; b) analisar o conteúdo dos artigos quanto a seus objetivos, metodologia empregada e principais resultados alcançados; e, c) sintetizar as principais contribuições das publicações em relação ao tema estudado, buscando delinear perspectivas de intervenção e pesquisas futuras.

\section{Resultados e discussão}

\section{Descrição geral dos artigos selecionados}

A busca bibliográfica na base de dados MedLine apresentou um total de 29 resultados que, após seleção segundo os critérios definidos neste estudo, foram reduzidos a cinco artigos. A busca empreendida na base de dados PsycINFO retornou 58 resultados, dos quais 11 foram qualificados para este estudo. A busca na base de dados Lilacs retornou um único resultado, que não se enquadrou nos critérios presentes de inclusão. Dos 16 artigos qualificados, três estavam duplicados, pois apareceram em duas bases de dados; dois artigos foram excluídos posteriormente, por terem como foco principal grupos de discussão online sustentados por comunidades virtuais. Restaram, portanto, 11 artigos que foram detalhadamente analisados.

O Quadro 1 apresenta a caracterização geral dos artigos selecionados. Nota-se, preliminarmente, que seis das 11 publicações são de origem norte-americana e que todas foram redigidas em língua inglesa. Os demais artigos são oriundos da Europa ou Austrália. Não foi encontrado nenhum artigo brasileiro ou oriundo da América Latina. Isso parece indicar que as pesquisas sobre os fatores psicológicos e psicossociais envolvidos no enfrentamento do câncer de próstata, que tenham como fundamento operacional os grupos de apoio, ainda são incipientes nos países latino-americanos.

Embora o intervalo da pesquisa tenha abarcado duas décadas, apenas um artigo ultrapassou o período dos últimos 10 anos, indicando que a preocupação com o tema é relativamente recente na literatura e, possivelmente, acompanha o aumento da incidência do câncer de prós- tata registrada em estudos epidemiológicos recentes $^{2}$. A revista Psycho-oncology aparece como periódico de destaque, uma vez que cinco dos 11 artigos selecionados para este estudo foram publicados nesse veículo. Dentre os demais artigos, apenas dois foram publicados em revistas da área médica ou de enfermagem (Journal of Urology e Urology Nursing). Esta constatação sugere que os profissionais de campos interdisciplinares são, provavelmente, os maiores interessados na investigação do uso de grupos como estratégia de tratamento psicológico de pacientes com câncer de próstata.

Quanto ao tipo de estudo e à estratégia metodológica utilizada, nota-se certa equivalência entre o número de estudos qualitativos (cinco) e quantitativos (seis), com predomínio de estudos descritivos e transversais. Quando se leva em conta os objetivos dessas pesquisas, identificou-se que os estudos quantitativos dedicam-se, sobretudo, a delinear o perfil sociodemográfico da população que participa de grupos de apoio aos pacientes com câncer de próstata, identificar os fatores que motivam essa participação, avaliar os benefícios advindos dessa modalidade de cuidado e delimitar as configurações grupais mais eficazes. Os estudos qualitativos visaram examinar a experiência de participar de um grupo de apoio na perspectiva dos pacientes com câncer de próstata e de suas esposas, refletir sobre como a sexualidade e as questões de gênero emergem dentro desses grupos, apresentar um modelo específico de grupo e explorar as implicações da doença e do adoecimento na construção das narrativas de vida dos homens afetados. Dentre esses estudos é possível destacar um, que apresenta uma metodologia inovadora ao sustentar um relato fictício de um paciente com câncer de próstata produzido a partir de observações que o autor fez em um grupo de apoio para homens acometidos.

\section{Principais contribuições dos artigos selecionados}

Tomando em consideração a ampla distribuição dos grupos de apoio como estratégia de cuidado em saúde, é possível indagar quais seriam as características da população que tende a se beneficiar dessa modalidade de atendimento psicológico. Um estudo ${ }^{7}$ dedicou-se a identificar os padrões de procura por essa modalidade de cuidado, tentando definir quais são as doenças e as características pessoais que mais favoreciam a adesão. Os resultados indicam que as doenças que determinam maior estigma social, como o 
câncer e a AIDS, favorecem a adesão aos grupos de apoio, em contraste com patologias graves, porém com menor estigma, como, por exemplo, as afecções cardíacas. O isolamento social, a deficiência da rede de apoio social e níveis mais baixos de escolaridade também favorecem a participação nesses grupos ${ }^{8}$.

Estes resultados parecem complementar os apresentados em outro estudo ${ }^{12}$, segundo o qual pacientes que moram em grandes centros urbanos são mais propensos a procurar ajuda em grupos de apoio. A hipótese apresentada pelos autores é que viver em grandes centros em geral deter- mina certo enfraquecimento da rede social e o esgarçamento das relações afetivas. Vistos em seu conjunto, tais estudos ${ }^{7,8,12}$ sugerem que os grupos de apoio funcionam como uma estratégia complementar de cuidado, cuja principal característica é oferecer um contexto de socialização e compartilhamento de sentimentos e emoções, ambos necessários para o enfrentamento de uma doença grave, que carrega o estigma de ser potencialmente fatal. Por ser uma patologia ainda muito discriminada e revestida de certos tabus na cultura ocidental, a vivência do câncer de próstata pode determinar o rompimento de relações soci-

Quadro 1. Apresentação das principais características dos artigos que compõem o corpus deste estudo.

\begin{tabular}{|c|c|c|c|c|c|c|}
\hline & Título do artigo & $\begin{array}{l}\text { País de } \\
\text { origem }\end{array}$ & $\begin{array}{c}\text { Ano de } \\
\text { publicação }\end{array}$ & $\begin{array}{l}\text { Base de } \\
\text { dados }\end{array}$ & Revista & $\begin{array}{l}\text { Tipo de } \\
\text { estudo }\end{array}$ \\
\hline 1 & $\begin{array}{l}\text { Breast cancer and prostate cancer self-help } \\
\text { groups }\end{array}$ & Canadá & 1996 & PsycINFO & Psycho-oncology & $\begin{array}{l}\text { Qualitativo, } \\
\text { descritivo }\end{array}$ \\
\hline 2 & $\begin{array}{l}\text { Sexuality, society and senior citizens: an } \\
\text { analysis of sex talk among prostate cancer } \\
\text { support group members }\end{array}$ & EUA & 2000 & PsycINFO & $\begin{array}{l}\text { Sexuality and Culture: } \\
\text { an Interdisciplinary } \\
\text { Quaterly }\end{array}$ & $\begin{array}{l}\text { Qualitativo, } \\
\text { descritivo }\end{array}$ \\
\hline 3 & $\begin{array}{l}\text { Thinking inside the box: on identity, sexuality, } \\
\text { prostate cancer and social support }\end{array}$ & EUA & 2000 & PsycINFO & $\begin{array}{l}\text { Journal of Aging and } \\
\text { Identity }\end{array}$ & $\begin{array}{l}\text { Qualitativo, } \\
\text { descritivo }\end{array}$ \\
\hline 4 & $\begin{array}{l}\text { Who talks? The social psychology of illness } \\
\text { support groups }\end{array}$ & EUA & 2000 & $\begin{array}{l}\text { PsycINFO } \\
\text { MedLine }\end{array}$ & American Psychology & $\begin{array}{l}\text { Quantitativo, } \\
\text { descritivo }\end{array}$ \\
\hline 5 & $\begin{array}{l}\text { The supportive care needs of men with } \\
\text { prostate cancer }\end{array}$ & Austrália & 2001 & PsycINFO & Psycho-oncology & $\begin{array}{l}\text { Quantitativo, } \\
\text { descritivo }\end{array}$ \\
\hline 6 & $\begin{array}{l}\text { Sociodemographic characteristics and health } \\
\text { related quality of life in men attending } \\
\text { prostate cancer support groups }\end{array}$ & EUA & 2002 & MedLine & Journal of Urology & $\begin{array}{l}\text { Quantitativo, } \\
\text { descritivo }\end{array}$ \\
\hline 7 & $\begin{array}{l}\text { Improving quality of life in men with prostate } \\
\text { cancer: a randomized controled trial of group } \\
\text { education intervention }\end{array}$ & EUA & 2003 & PsycINFO & Health Psychology & $\begin{array}{l}\text { Quantitativo, } \\
\text { experimental }\end{array}$ \\
\hline 8 & $\begin{array}{l}\text { Shared decision making in patients with newly } \\
\text { diagnosed prostate cancer: a model for } \\
\text { treatment education and support }\end{array}$ & EUA & 2004 & MedLine & Urology Nursing & $\begin{array}{l}\text { Qualitativo, } \\
\text { descritivo }\end{array}$ \\
\hline 9 & $\begin{array}{l}\text { Determinants of participation in social } \\
\text { support groups for prostate cancer patients }\end{array}$ & Holanda & 2007 & $\begin{array}{l}\text { MedLine } \\
\text { PsycINFO }\end{array}$ & Psycho-oncology & $\begin{array}{l}\text { Quantitativo, } \\
\text { descritivo }\end{array}$ \\
\hline 10 & $\begin{array}{l}\text { What is the ideal support group? Views of } \\
\text { Australian people with cancer and their careers }\end{array}$ & Austrália & 2007 & $\begin{array}{l}\text { PsycINFO } \\
\text { MedLine }\end{array}$ & Psycho-oncology & $\begin{array}{l}\text { Quantitativo, } \\
\text { descritivo }\end{array}$ \\
\hline 11 & $\begin{array}{l}\text { Women and prostate cancer support groups: } \\
\text { The gender connect? }\end{array}$ & Canadá & 2008 & PsycINFO & $\begin{array}{l}\text { Social Science and } \\
\text { Medicine }\end{array}$ & $\begin{array}{l}\text { Qualitativo, } \\
\text { etnográfico }\end{array}$ \\
\hline
\end{tabular}


ais importantes e necessárias para o bem-estar psicossocial do paciente acometido. O isolamento social pode ser ainda maior se considerarmos as restrições que o próprio tratamento impõe ao paciente. Muitas vezes, pacientes que se submetem à prostatectomia radical apresentam sequelas com alto potencial de limitação da vida social, como incontinência urinária e impotência sexual.

Desse modo, pode-se afirmar que um dos principais benefícios dos grupos de apoio a pacientes que enfrentam o câncer de próstata é a possibilidade de manutenção e/ou criação de uma rede de apoio psicossocial, que é considerada fundamental para o bom enfrentamento da doença. Estudos ${ }^{13,14}$ destacam ainda que os homens tendem a procurar pelos grupos de apoio em momentos mais críticos da doença, ou seja, na ocasião do diagnóstico e no agravamento da enfermidade. Reforçam, assim, o papel do grupo como espaço de amparo às angústias despertadas ao longo dos diferentes estágios de enfrentamento da doença, do diagnóstico ao tratamento e reabilitação psicossocial.

Outra característica apontada como preditora de adesão aos grupos de apoio foi a atitude positiva do paciente em relação à participação em grupos ${ }^{7}$. Este achado fornece uma pista valiosa para que essa predisposição seja investigada minuciosamente junto aos pacientes, antes de se fazer a indicação ao grupo.

Quanto aos potenciais benefícios advindos da participação nos grupos, os estudos destacam o aumento no nível geral de informação sobre a doença, melhor adaptação às sequelas físicas do câncer e do tratamento, atitudes mais positivas frente aos cuidados com a saúde, maior nível de ajustamento sexual, maior adesão ao tratamento, melhor relacionamento com o cônjuge e menor preocupação com a incontinência urinária ${ }^{14,15}$. Para as esposas de homens com câncer de próstata, a participação nos grupos permitiu a criação de uma rede de apoio com outras mulheres, por meio da qual puderam obter mais informações acerca da doença de seu companheiro, fortalecer o sentimento de esperança e contar com asseguramento quanto à maneira de ajudar o parceiro ${ }^{16}$. Os autores destacam que mulheres tendem a se engajar nos grupos de apoio na busca por um espaço de compartilhamento de emoções, o que corresponderia à construção dos papéis e da identidade de gênero feminino. Durante os grupos, as mulheres tendem a assumir três papéis fundamentais: cossobrevivente, facilitadora social e apoiadora. Estes estariam vinculados à construção do "ser mulher" na sociedade oci- dental como principal responsável pelo provimento de cuidados básicos em saúde, nutrição e socialização.

Nesse sentido, quando se comparam as motivações de homens com câncer de próstata e de mulheres com câncer de mama para participar de grupos de apoio, nota-se que os homens tendem a enfatizar a dimensão informativa do grupo, enquanto que as mulheres destacam o aspecto afetivo das interações estabelecidas ${ }^{17}$. Os homens tendem a valorizar o conhecimento de um expert, como, por exemplo, um profissional da saúde, enquanto as mulheres atribuem maior importância à contribuição dos pares. Para os homens é importante incluir a família no processo terapêutico, em contraste com a postura mais isolada das mulheres, que preferem estar somente com outras afetadas pela doença.

Quanto aos temas abordados nos dois grupos, constata-se que os homens têm mais facilidade para abordar questões ligadas à sexualidade, enquanto que as mulheres demonstram maior necessidade em falar da morte. Conforme apontado no estudo, a vivência de um câncer que atinge órgãos simbolicamente associados à identidade sexual de homens e mulheres é atravessada por questões de gênero, que se concretizam na produção daquilo que socialmente é reconhecido como próprio ao masculino e ao feminino ${ }^{17}$. Assim, a facilidade dos homens em falar abertamente de sexualidade, em contraponto com o silêncio frente às questões ligadas à finitude, sustentamse em uma masculinidade socialmente construída como potente e viril. Neste modo hegemônico de "ser homem" na cultura ocidental, não há espaço para se confrontar a perspectiva concreta da morte. A preocupação crucial do homem acometido pelo câncer de próstata é com a possibilidade das sequelas, principalmente de ficar impotente em decorrência do tratamento cirúrgico, o que pode afastá-lo de outras preocupações igualmente relevantes, que dizem respeito à adesão ao tratamento e à melhoria da qualidade de vida. Este modo particular de apreensão da experiência do adoecimento traz uma importante dimensão a ser considerada nas pesquisas e nas intervenções direcionadas aos homens acometidos.

Quando se leva em conta o impacto do câncer de próstata sobre a vivência da sexualidade e a construção da masculinidade para os homens afetados, nota-se que o enfrentamento da doença lhes determina uma reconfiguração das noções de sexualidade ${ }^{18}$. Para os homens que enfrentam o câncer de próstata, em geral, a relação sexual era definida, antes do aparecimento da doença, 
por seu caráter espontâneo e pela penetração do pênis na vagina. Ao participar de um grupo de apoio, os homens estudados se engajaram em um processo de redefinição dos significados atribuídos à sexualidade e à atividade sexual, sobretudo no que tange a esses dois construtos. Nesse contexto, o grupo constitui-se como importante espaço para negociação de significados para a relação sexual, as formas de intimidade e a abstinência sexual. Em outra investigação, o mesmo autor aponta ainda para ampla negociação da própria identidade a que os homens se lançam a partir do momento em que iniciam o enfrentamento do câncer de próstata ${ }^{19}$. Parte dessa negociação inclui a reconfiguração da relação conjugal e a criação de uma nova identidade social.

Dentro do cenário delineado pela literatura analisada neste estudo, é possível traçar algumas considerações sobre propostas de intervenção em grupo voltadas para atender as necessidades dessa população e apresentar alguns modelos que parecem ser mais apropriados. Um estudo ${ }^{8}$ apresenta um modelo de tratamento no qual o grupo de apoio é parte integrante de um programa mais amplo, que auxilia o paciente e seus familiares nos processos de tomada de decisão sobre o tratamento. Nesse modelo, os profissionais de saúde se esforçam para colocar o paciente em uma postura ativa e responsável por buscar sua melhora. Para tanto, são organizados grupos informativos, que têm como objetivo fornecer subsídios para o acolhimento emocional, contenção da angústia, provimento de informação e espaços de escuta e discussão. Esses grupos são oferecidos para o paciente, bem como para seus familiares e cuidadores. O coordenador desse grupo centra-se em quatro aspectos básicos: estabelecer um rapport, explorar as preocupações dominantes entre os participantes, estabelecer uma comunicação efetiva e favorecer ações de saúde. Segundo os autores, tal modelo de intervenção aumenta a satisfação, tanto do paciente como do clínico, com o tratamento. Os pacientes apresentam melhor adesão ao tratamento e desenvolvem melhores recursos para autocuidado em saúde.

Os resultados de um estudo que investigou qual seria o modelo ideal de grupo de apoio para homens revelou que os participantes valorizam o aspecto homogêneo do grupo, a afetividade do coordenador e a possibilidade de obter informação sobre a doença ${ }^{20}$. Para os homens, o grupo é um espaço no qual podem desenvolver o senso de pertencimento à comunidade, aceitação incondicional e empatia. Os participantes do estudo demonstraram apreço em ter um coordenador que também enfrentava o câncer de próstata e consideravam indispensável que ele tivesse algum treinamento prévio na condução e na coordenação de grupos. A participação de homens em diferentes momentos do enfrentamento da doença e com diferentes prognósticos foi positivamente considerada pelos participantes. Assim, o estudo sugere que os grupos de apoio a homens com câncer de próstata devem considerar: as necessidades específicas da população atendida, a avaliação contínua do nível de satisfação dos participantes, a inclusão de pacientes com diferentes prognósticos, o modelo de coordenação participativa, o treino contínuo e a supervisão dos coordenadores, a oferta ativa de informações, bem como a inclusão e o intercâmbio com diferentes profissionais de saúde.

De modo geral, a literatura sugere ainda que o coordenador do grupo seja sensível e treinado para abordar questões de sexualidade com os participantes, uma vez que tais preocupações podem ficar encobertas se o coordenador não tiver uma postura aberta e não-preconceituosa ${ }^{18}$. Quanto ao envolvimento nos grupos, os estudos afirmam que os profissionais de saúde têm papel fundamental na estimulação à participação dos pacientes nessa modalidade de intervenção, bem como de suas esposas ${ }^{7,16}$.

\section{Considerações finais}

A revisão integrativa da literatura disponível sobre grupos de apoio para homens com câncer de próstata permite traçar algumas considerações sobre a pesquisa científica que se opera nesse campo. A investigação dos grupos de apoio psicológico para esses pacientes afigura-se como uma ferramenta importante que permite ao profissional de saúde compreender as condições que motivam a participação da população em tais grupos e os fatores terapêuticos que legitimam essa estratégia de cuidado dentre outras opções de atenção em saúde, além de fornecer subsídios para planejamento de sua implantação e condução em diferentes contextos.

Por outro lado, esta revisão possibilita dimensionar algumas limitações nesse campo. Em primeiro lugar destaca-se a inexistência de trabalhos latino-americanos e brasileiros indexados nas bases bibliográficas consultadas. Questiona-se se essa inexistência deve-se ao pouco interesse que essa temática desperta na comunidade científica, à ausência de grupos organizados (a exemplo dos norteamericanos US-TOO e Men-to-Men) ou até mes- 
mo ao modelo hegemônico de construção da masculinidade no contexto latino-americano.

Esta constatação ganha especial relevância se considerarmos o cenário delineado pelas políticas de saúde pública no Brasil, sobretudo àquelas definidas nas diretrizes da Política Nacional de Atenção Integral à Saúde do Homem6 . Segundo estas diretrizes, a atenção à saúde do homem deve priorizar a atenção básica, com enfoque especial às ações coordenadas pelo Programa de Saúde da Família, no qual a estratégia de grupo tem sido amplamente utilizada. Assim, pode-se considerar que a implantação e o incremento de atividades grupais destinados à população masculina na atenção básica possivelmente tomará como referência estudos realizados em contextos culturais bastante diversos. Neste sentido, recomenda-se tanto que a utilização destes estudos ocorra de modo crítico e que esforços científicos sejam feitos de modo a compreender as especificidades do contexto brasileiro.

Em segundo lugar, vale destacar que os estudos dedicam-se pouco à caracterização dos grupos quanto ao seu formato e à abordagem teórica e técnica. Nenhum dos estudos analisados apresentou uma descrição detalhada e sistematizada de como o coordenador organizava suas intervenções e qual era o enfoque psicológico que utilizava para a compreensão dos fenômenos grupais.
Por fim, considera-se que as evidências disponíveis oferecem ampla sustentação empírica para que se afirme a aplicabilidade dos grupos de apoio psicológico no acompanhamento de homens com câncer de próstata. O conhecimento aqui sistematizado tem impacto na formulação de estratégias de atenção à saúde do homem no contexto da saúde pública, pois auxilia na definição de uma modalidade de cuidado adequada a esta população e que se articula com a atual política de saúde pública destinada a esta população. Segundo esta política, os serviços de saúde devem se empenhar em expandir a participação masculina nos serviços, trabalhando de modo a incluir o enfoque de gênero. Como visto, os grupos de apoio são estratégias de cuidado que favorecem o trabalho nestas duas direções.

Contudo, ainda são necessários estudos futuros que se dediquem a dimensões pouco exploradas desses grupos, como a operacionalização das intervenções dos coordenadores, a comparação entre diferentes abordagens teóricas e a determinação mais precisa do potencial terapêutico e dos limites de aplicação dessa modalidade de tratamento. De um modo geral, as pesquisas empreendidas até o momento salientam a importância de se implementar grupos como estratégia complementar de cuidado com homens que enfrentam o câncer de próstata.

\section{Colaboradores}

MS Moscheta trabalhou na concepção e no delineamento do estudo, na pesquisa e no levantamento de material bibliográfico, na análise dos dados, na redação e revisão crítica do artigo e na aprovação de sua versão final. MA Santos trabalhou no delineamento do estudo, na análise dos dados, na redação e revisão crítica do artigo e na aprovação de sua versão final. 


\section{Referências}

1. Guerra MR, Gallo CVM, Mendonça GAS. Risco de câncer no Brasil: tendências e estudos epidemiológicos mais recentes. Rev Bras Cancerologia 2005; 51(3):227-234.

2. Brasil. Ministério da Saúde. Estimativa 2010: incidência de câncer no Brasil. Rio de Janeiro: Instituto Nacional do Câncer; 2009.

3. Dini L, Koff WJ. Perfil do câncer de próstata no hospital de clínicas de Porto Alegre. Rev Assoc Med Bras 2006; 52(1):34-56.

4. Gomes R, Rebello LEFS, Araújo FC, Nascimento EF. A prevenção do câncer de próstata: uma revisão da literatura. Cien Saude Colet 2008; 13(1):235246.

5. Gomes R, Nascimento EF, Rebello LEFS, Araújo FC. As arranhaduras da masculinidade: uma discussão sobre o toque retal como medida de prevenção do câncer prostático. Cien Saude Colet 2008; 13(6):1975-1984.

6. Brasil. Ministério da Saúde. Política nacional de atenção integral à saúde do homem: princípios e diretrizes. [texto na internet]. 2008 [acessado $2009 \mathrm{dez} 14$ ]. [cerca de 46p.]. Disponível em: http://dtr2001. saude.gov.br/sas/PORTARIAS/Port2008/PT-09CONS.pdf

7. Voerman B, Visser A, Fischer M, Garssen B, Van Andel G, Bensing J. Determinants of participation in social support groups for prostate cancer patients. Psychooncology 2007; 16(12):1092-1099.

8. Colella KM, Deluca G. Shared decision making in patients with newly diagnosed prostate câncer: a model for treatment education and support. Urol Nurs 2004; 24(3):187-191.

9. Broome ME. Integrative literature reviews in the development of concepts. In: Rodgers BL, Knafl $\mathrm{KA}$, organizadores. Concept development in nursing: foundations, techniques and applications. Philadelphia: W.B. Saunders; 1993. p.193-215.

10. Mendes KDS, Silveira RCCP, Galvão CM. Revisão integrativa: método de pesquisa para a incorporação de evidências na saúde e na enfermagem. Texto-Contexto Enferm 2008; 17(4):758-764.

11. Ganong LH. Integrative reviews of nursing research. Res Nurs Health 1987; 10(1):1-11.

12. Davison KP, Pennebaker JW, Dickerson SS. Who talks? The social psychology of illness support groups. Am Psychol 2000; 55(2):205-217.
13. Steginga SK, Occhipinti S, Dunn J, Gardiner RA, Heathcote P, Yaxley J. The supportive care needs of men with prostate cancer. Psychooncology 2001; 10(1):66-75.

14. Katz D, Koppie TM, Wu D, Meng MV, Grossfeld GD, Sadesky N, Lubeck DP, Carroll PR. Sociodemographic characteristics and health related quality of life in men attending prostate cancer support groups. J Urol 2002; 168(5):2092-2096.

15. Lepore SJ, Helgeson VS, Eton DT, Schulz R. Improving quality of life in men with prostate cancer: a randomized controlled trial of group education interventions. Health Psychol 2003; 22(5):443-452.

16. Bottorff JL, Oliffe JL, Hapin M, Phillips M, McLean G, Mroz L. Women and prostate cancer support groups: the gender connect? Soc Sci Med 2008; 66(5): 1217-1227.

17. Gray R, Fitch M, Davis C, Phillips C. Breast cancer and prostate cancer self-help groups: reflections on differences. Psychooncology 1996; 5(2):137-142.

18. Arrington M. Sexuality, society, and senior citizens: an analysis of sex talk among prostate cancer support group members. Sex Cult 2000; 4(4):45-74.

19. Arrington M. Thinking inside the box: on identity, sexuality, prostate cancer, and social support. $J \mathrm{Ag}$ ing Ident 2000; 5(3):151-158.

20. Butow PN, Kirsten LT, Ussher JM, Wain, GV, Sandoval M, Hobbs KM, Hodgkinson K, Stenlake A. What is the ideal support group? Views of Australian people with cancer and their carers. Psychooncology 2007; 16(11):1039-1045.

Artigo apresentado em 13/01/2011

Aprovado em 25/03/2011

Versão final apresentada em 04/04/2011 